\title{
The number of metastatic lymph nodes exhibiting poorly differentiated clusters predicts survival in patients with pStage III colorectal cancer
}

\author{
Osamu Kinoshita $^{1,2}$ - Mitsuo Kishimoto ${ }^{1}$ - Yasutoshi Murayama ${ }^{2}$ - Yoshiaki Kuriu ${ }^{2}$. \\ Masayoshi Nakanishi $^{2} \cdot$ Chohei Sakakura $^{2} \cdot$ Eigo Otsuji $^{2} \cdot$ Akio Yanagisawa $^{1}$
}

Accepted: 17 September 2015 / Published online: 28 September 2015

(C) The Author(s) 2015. This article is published with open access at Springerlink.com

\begin{abstract}
Purpose Many studies have proposed alternative designations for lymph node (LN) status in colorectal cancer (CRC); however, knowledge of histopathological features in metastatic lymph nodes (MLNs) is limited. This study investigated the clinicopathological significance of poorly differentiated clusters (PDCs) in MLNs.

Methods Slides from 159 patients with pathological Stage III CRC were reviewed. Those with $<12$ dissected LNs (DLNs) were ineligible. PDCs composed of $\geq 5$ cancer cells lacking full glandular formation and $\geq 10$ PDCs under $\times 20$ objective lens were defined as positive, and the number of MLNs with positive PDCs (MLNs-PDCs) was counted. Results were correlated with patient survival and comparisons made with other indications of LN status.

Results The mean numbers of MLNs and MLNs-PDCs were 2.8 and 1.0, respectively, and were moderately and positively correlated with each other. Univariate analysis identified cutoffs of $\geq 5$ MLNs ( 86 vs. $55 \%, p=0.024$ ), $\geq 2$ MLNs-PDCs ( 85 vs. $63 \%, p=0.008$ ), and $\geq 30 \% \mathrm{LN}$ ratio ( 85 vs. $44 \%, p=$ 0.036 ) to indicate a positive LN status. However, no cutoff for DLNs was obtained. MLNs-PDCs $(\geq 2)$ were associated with pT4 tumor ( $p=0.0035)$, open surgery $(p=0.016)$, greater number of MLNs $(p<0.0001)$, and positive-PDC primary tumor $(p<0.0001)$. In multivariate analysis, a prognostic model
\end{abstract}

Mitsuo Kishimoto

kish-pat@koto.kpu-m.ac.jp

1 Department of Surgical Pathology, Kyoto Prefectural University of Medicine, 465 Kajii-cho, Kamigyo-ku, Kyoto 602-8566, Japan

2 Department of Surgery, Division of Digestive Surgery, Kyoto Prefectural University of Medicine, 465 Kajii-cho, Kamigyo-ku, Kyoto 602-8566, Japan incorporating $\geq 2$ MLNs-PDCs provided the lowest Akaike information criterion value; consequently, both pT4 tumors $(p<0.001)$ and $\geq 2$ MLNs-PDCs $(p=0.038)$ were revealed to be significant prognosticators.

Conclusion Results showed that applying the number of MLNs-PDCs could improve stratification in pStage III CRC and may be a valuable candidate for LN status.

Keywords Colorectal cancer $\cdot$ Lymph node status $\cdot$ Poorly differentiated clusters $\cdot$ Histopathological prognosticator

\section{Introduction}

The incidence of colorectal cancer (CRC) has increased gradually over the decades in developed countries, and CRC now is the third most-commonly diagnosed cancer [1]. Recent progress in active cytotoxic chemotherapy regimens has contributed to prolonged survival of patients with CRC $[2,3]$. However, to reduce mortality, there is a growing need for early prediction of prognosis to further improve postoperative interventions.

Since Dukes [4] reported the histopathological grading of rectal cancer in 1937, accumulated evidence on CRC has confirmed the clinical importance of lymph node (LN) status. In particular, the number of metastatic LNs (MLNs) has been considered one of the most powerful predictors in CRC [5, 6] and guides the indication for adjuvant chemotherapy. On the other hand, many researchers have proposed alternatives to indicate LN status, including the total number of dissected LNs (DLNs) [7-10], LN ratio (LNR) [11-15], and extramural cancer deposits (EX) [16, 17]. However, to date, published studies rarely have focused on the histopathological characteristics of MLNs. 
More recently, a novel histological grading system using poorly differentiated clusters (PDCs), which are composed of $\geq 5$ cancer cells that present at the invasive front of the tumor and lack full glandular formation, has been highlighted as a histopathological prognosticator of CRC [18-20]. Presence of PDCs was reported to be strongly positively correlated with LN metastasis and poorer survival and, consequently, was expected to provide a complementary approach to the conventional pathological diagnostic process. However, despite increasing evidence of PDCs as prognostic indicators, no previous studies have focused on PDCs in MLNs and their correlation to PDCs in primary tumors. An understanding of the histopathological behavior of PDCs in MLNs may provide insights into the key process following $\mathrm{LN}$ metastasis. Therefore, this study expanded upon the PDC concept of evaluation for histopathological features of MLNs and aimed to clarify the clinicopathological importance of PDCs in MLNs in comparison with previously reported LN status.

\section{Patients and methods}

\section{Patients}

This study examined data on patients with pathological Stage III (pStage III) CRC who had undergone potentially curative resection and systematic en bloc $\mathrm{LN}$ dissection at a single institution from 2000 to 2012. Since laparoscopic surgery became widespread during that period, cases in this study were treated with either open or laparoscopic surgery. Following the recommendation of the Union for International Cancer Control (UICC) TNM classification [5] to eliminate possible staging migration, patients with $<12$ DLNs were ineligible for this study. In addition, although pelvic sidewall LN dissection, which means removal of internal iliac and obturator LNs, traditionally has been performed in Japan for locally advanced lower rectal cancer [21,22], patients on whom that dissection method had been performed were excluded. Other exclusion criteria for this retrospective cohort included (1) synchronous or multiple cancers, (2) preoperative therapy (neoadjuvant chemotherapy and endoscopic resection), (3) cancers related to ulcerative colitis or Crohn's disease, and (4) familial adenomatous polyposis or hereditary non-polyposis colorectal cancer. To be included for analysis, after resection, patients were required to have follow-up surveillance at 3-month intervals for 1 year, then every 6 months for 3 years, and yearly thereafter. Prior to the study's initiation, approval to use patient data for research purposes was obtained at the institution.

\section{Specimens}

Within an hour after resection, all regional LNs were manually retrieved from the en bloc resected specimens after visual observation and palpation. The process of sectioning specimens after formalin fixation was performed according to Japanese guidelines [6]. In particular, larger retrieved LNs were longitudinally cut through the hilus, and smaller ones were embedded in paraffin whole. Sections $3 \mu \mathrm{m}$ thick were cut from paraffin-embedded specimens and mounted onto glass slides. After deparaffinization, slides were stained with conventional hematoxylin and eosin according to the standard procedure.

\section{Histopathological evaluation}

According to criteria set previously [18, 19], PDCs were defined as cancer clusters lacking full glandular formation and composed of $\geq 5$ cancer cells, which are commonly observed at the invasive front of the tumor. All slides were reviewed pathologically, with special attention paid to the presence of PDCs, and $\geq 10$ PDCs under a microscopic field with a $\times 20$ objective lens were defined as positive PDCs. Our previous study [23] reported representative slides of PDCs in primary tumors. In the present study, PDCs in primary tumors were evaluated at the invasive front, whereas PDCs in MLNs were evaluated where they were observed most commonly (Fig. 1), and the number of MLNs with positive PDCs (MLNs-PDCs) was counted.

In addition to a pathologically proven number of DLNs, the LNR, defined as the number of MLNs divided by the number of DLNs, was assessed as LN status. For EX lacking residual LN structure, the study adopted the definition in Japanese guidelines [6]; consequently, EX were comprehensively considered as MLNs. However, tumor nodules that might be related to an isolated cancer focus in the lymphovascular vessels or perineural space were not considered as MLNs [16].

In addition, other pathological variables were obtained by observing slides. All slides were evaluated by a trained pathologist specializing in gastrointestinal pathology and blinded to the patients' outcome.

\section{Statistical analysis}

Overall survival (OS) curves were drawn by the Kaplan-Meier method, and comparisons between groups were performed using the log-rank test, with the length of the follow-up period being truncated at 5 years. In addition, the cutoff for each LN status was optimized by maximizing the significance. Correlation analysis was performed using the Spearman rank correlation coefficient. Multivariate Cox proportional hazard regression analysis was developed using stepwise regression among representative variables, and the hazard radio (HR) and $95 \%$ confidence interval $(\mathrm{CI})$ for each variable were calculated. Furthermore, adequacy of the parametric model was assessed by the Akaike information criterion (AIC) [24] to estimate the most fitting model with the smallest information 

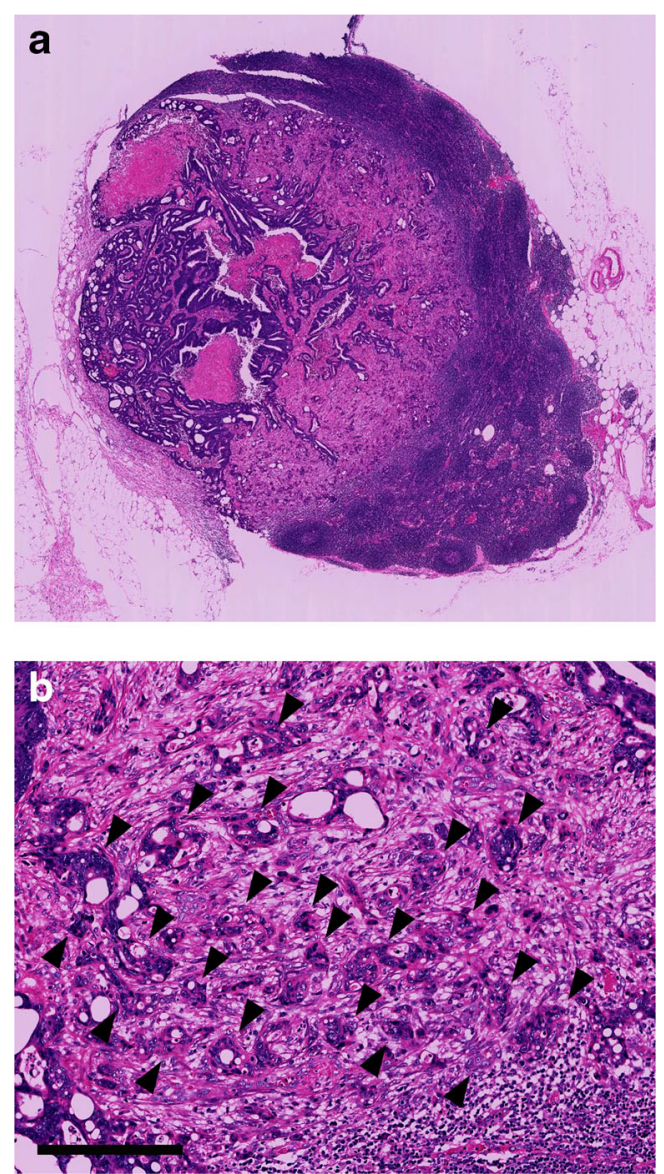

Fig. 1 Representative examples of metastatic lymph nodes with positive PDCs under low-power view (a) and under the view of $\times 20$ objective lens using hematoxylin and eosin stain (b). PDCs $(>10)$ are observed (arrowheads). The scale bar indicates $200 \mu \mathrm{m}$

loss and to compare models consisting of various combinations of variables. Differences were considered statistically significant at the $p<0.05$ level.

All statistical calculations were performed by EZR statistical software (Saitama Medical Center, Jichi Medical University, Saitama, Japan) [25], which is a graphical user interface for R (The R Foundation for Statistical Computing, Vienna, Austria).

\section{Results}

The study enrolled 159 patients. As Table 1 shows, 111 tumors $(70 \%)$ were colonic and $48(30 \%)$ were rectal. Most patients had pT3 or pT4 lesions ( $90 \%$ ), and half had positivePDC primary tumors $(50 \%)$. One hundred seven patients (69\%) received adjuvant chemotherapy consisting mainly of 5-fluorouracil (5-FU); however, 52 (31\%) did not. At the time of this study, the median follow-up was 48.5 months and the 5 -year OS rate of this cohort was $79 \%$. On average, there were 22.7 DLNs; of those, 2.8 MLNs and 1.0 MLN-PDCs
Table 1 Patients and tumor characteristics $(n=159)$

\begin{tabular}{|c|c|}
\hline \multicolumn{2}{|l|}{ Variables } \\
\hline Age (year) [mean (range, SD)] & $65.2(21-94,11.9)$ \\
\hline \multicolumn{2}{|l|}{$\operatorname{Sex}[n(\%)]$} \\
\hline Male & $76(55)$ \\
\hline Female & $83(45)$ \\
\hline \multicolumn{2}{|l|}{ Tumor location $[n(\%)]$} \\
\hline \multicolumn{2}{|l|}{ Colon } \\
\hline Cecum-transverse & $51(32)$ \\
\hline Descending-sigmoid & $60(38)$ \\
\hline Rectum & $48(30)$ \\
\hline Tumor size (mm) [mean (range, SD)] & $47(10-110,19.4)$ \\
\hline \multicolumn{2}{|l|}{ T status $[n(\%)]$} \\
\hline pT1 & $1(1)$ \\
\hline pT2 & $15(9)$ \\
\hline pT3 & $105(66)$ \\
\hline pT4 & $38(24)$ \\
\hline \multicolumn{2}{|l|}{ PDCs in primary tumor $[n(\%)]$} \\
\hline Positive & $80(50)$ \\
\hline Negative & $79(50)$ \\
\hline DLNs [mean (range, SD)] & $22.7(12-91,11.7)$ \\
\hline MLNs [mean (range, SD)] & $2.8(1-18,2.7)$ \\
\hline LNR [median (IQR)] & $0.09(0.06-0.17)$ \\
\hline MLNs-PDCs [mean (range, SD)] & $1.0(0-8,1.4)$ \\
\hline \multicolumn{2}{|l|}{ Operative approach $[n(\%)]$} \\
\hline Open & $83(52)$ \\
\hline Laparoscopic & $76(48)$ \\
\hline \multicolumn{2}{|l|}{ Adjuvant chemotherapy $[n(\%)]$} \\
\hline With & $107(69)$ \\
\hline Without & $52(31)$ \\
\hline
\end{tabular}

PDCs poorly differentiated clusters, $D L N s$ dissected lymph nodes, $M L N S$ metastatic lymph nodes, $L N R$ lymph node ratio, $M L N s-P D C s$ metastatic lymph nodes with positive PDCs

were detected pathologically. No difference was found between open and laparoscopic surgery in the number of DLNs and MLNs (mean DLNs, 23.5 vs. 21.7, respectively; mean MLNs, 3.1 vs. 2.6, respectively). The study found a moderately positive correlation between the number of MLNs-PDCs and number of MLNs and a slight correlation between the number of MLNs with positive and negative PDCs. Figure 2 shows scatter plots for the number of MLNs-PDCs and MLNs.

Based on the Kaplan-Meier survival method, univariate analysis identified a cutoff of 5 MLNs ( 86 vs. $55 \%, p=$ 0.024 ), 2 MLNs-PDCs ( 85 vs. $63 \%, p=0.008$ ), and LNR of $30 \%$ ( 85 vs. $44 \%, p=0.036$ ) as the statistically reliable stratifications to discriminate patient survival (Fig. 3). Given this, we decided upon $\geq 5$ MLNs, $\geq 2$ MLNs-PDCs, and $\geq 30 \%$ LNR to indicate positive LN status for subsequent analysis. On the other hand, when the study analyzed from 13 to 91 


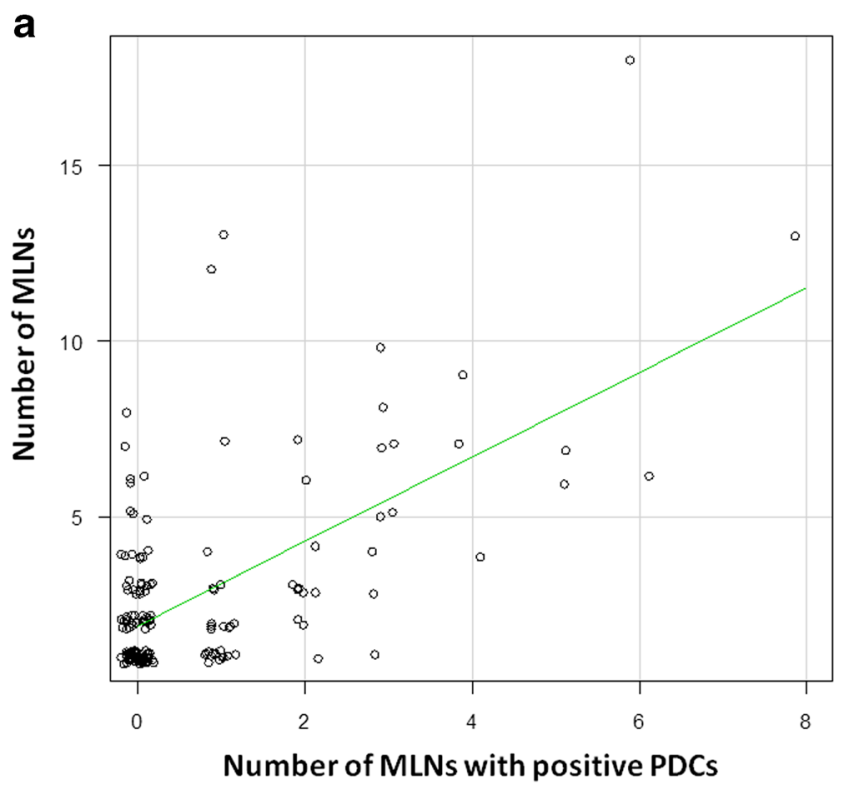

b

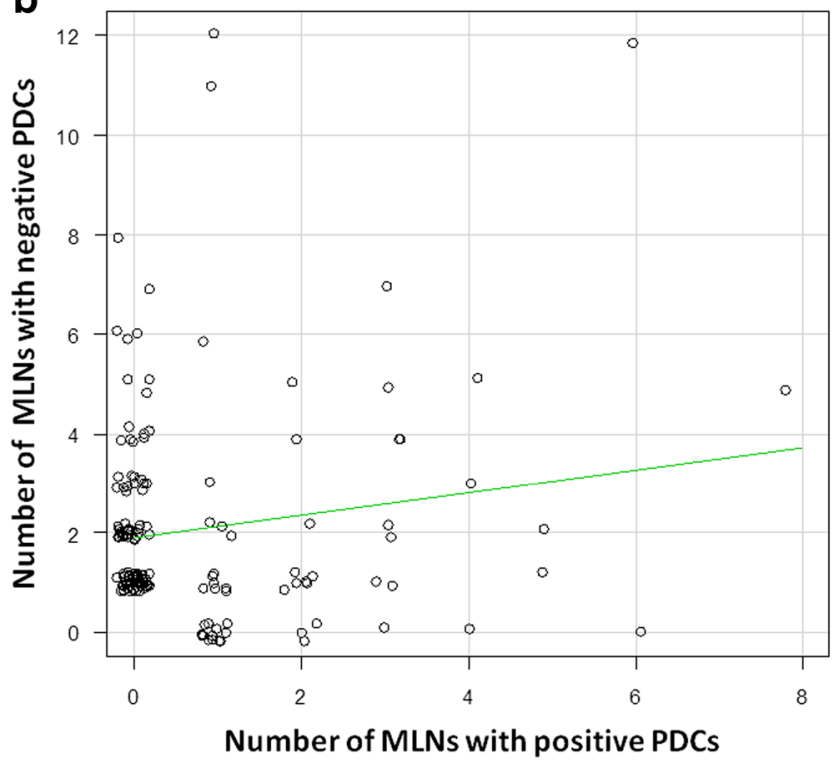

Fig. 2 a Scatter plots for the number of MLNs-PDCs and the number of MLNs. The graph shows a moderate correlation between the two (Spearman rank correlation coefficient $0.405, p<0.0001$ ). b Scatter plots for the number of MLNs with positive and negative PDCs. Little correlation is found between the two (Spearman rank correlation coefficient $0.145, p=0.068$ )

DLNs, a statistical threshold for 5-year OS was not obtained. Table 2 shows comparison of clinicopathological variables between patients with $\geq 2$ MLNs-PDCs and $<2$. MLNs-PDCs $(\geq 2)$ were associated with pT4 tumors $(p=$ $0.0035)$, open surgery $(p=0.016)$, a greater number of MLNs $(p<0.0001)$, and a positive-PDC primary tumor $(p<0.0001)$. When the combination of PDC status in primary tumors and the number of MLNs-PDCs was examined, there were only two (3\%) negative-PDC primary tumors among those with $\geq 2$ MLNs-PDCs. In contrast,
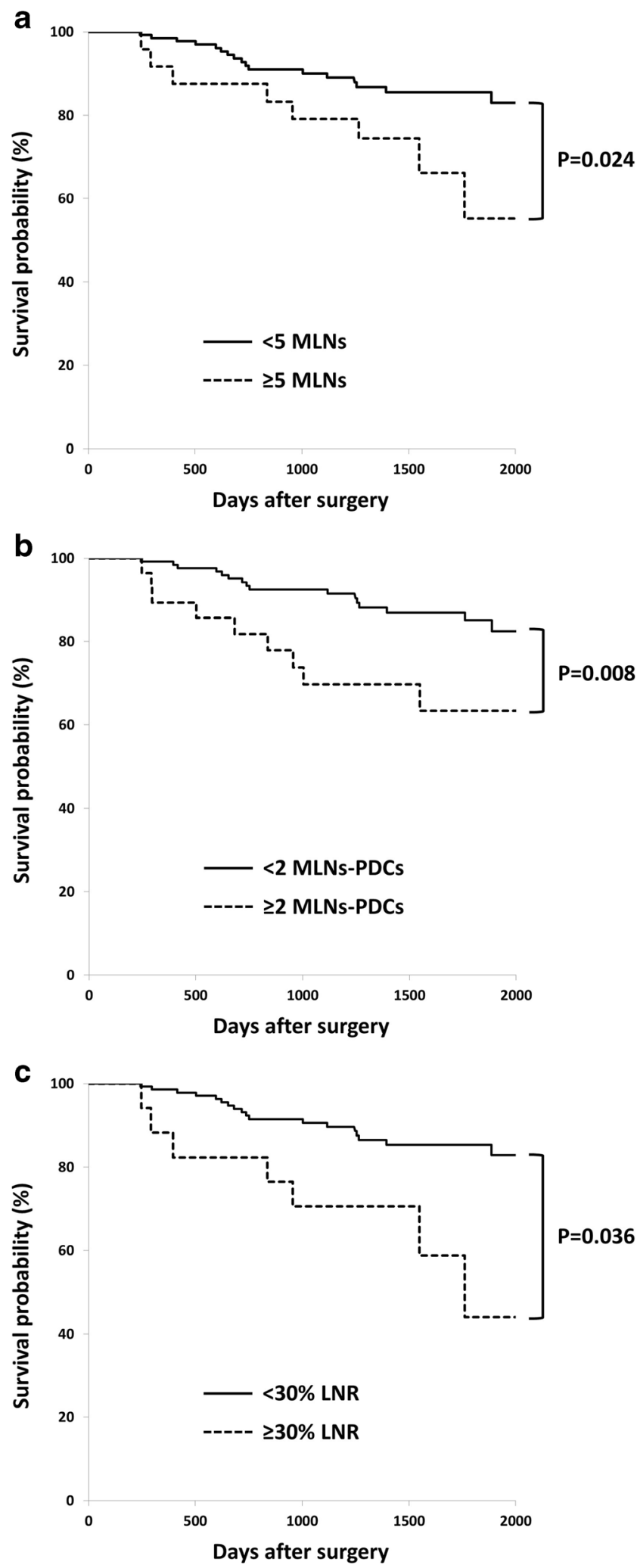

Fig. 3 Comparison of survival curves for each lymph node status

of patients with <2 MLNs-PDCs, 53 (41\%) had positive-PDC primary tumors and $77(59 \%)$ had negative-PDC ones. 
Table 2 Comparison of variables between patients with $\geq 2$ and $<2$ MLNs-PDCs

\begin{tabular}{|c|c|c|c|}
\hline Variables & $\begin{array}{l}\geq 2 \text { MLNs-PDCs } \\
(\mathrm{n}=29)\end{array}$ & $\begin{array}{l}<2 \text { MLNs-PDCs } \\
(n=130)\end{array}$ & $\mathrm{P}$ value \\
\hline Age (year) [mean (range, SD)] & $66.5(21-95,14.0)$ & $65.0(21-85,11.4)$ & \\
\hline \multicolumn{4}{|l|}{$\operatorname{Sex}[n(\%)]$} \\
\hline Male & $15(10)$ & $61(38)$ & \multirow[t]{2}{*}{0.64} \\
\hline Female & $14(9)$ & $69(43)$ & \\
\hline \multicolumn{4}{|l|}{ Tumor location $[n(\%)]$} \\
\hline Colon & & & \multirow[t]{3}{*}{0.11} \\
\hline Cecum-transverse & $12(8)$ & $39(25)$ & \\
\hline Descending-sigmoid & $6(4)$ & $54(34)$ & \\
\hline Rectum & $11(7)$ & $37(24)$ & \\
\hline Tumor size (mm) [mean (range, SD)] & $54(20-110,24.3)$ & $45(20-110,18.0)$ & 0.16 \\
\hline \multicolumn{4}{|l|}{ T status $[n(\%)]$} \\
\hline pT1 & $0(0)$ & $1(1)$ & \multirow{4}{*}{0.0035 (T1-3 vs. T4) } \\
\hline pT2 & $0(0)$ & $15(9)$ & \\
\hline pT3 & $16(10)$ & $89(56)$ & \\
\hline pT4 & $13(8)$ & $25(16)$ & \\
\hline \multicolumn{4}{|l|}{ PDCs in primary tumor $[n(\%)]$} \\
\hline Positive & $27(17)$ & $53(33)$ & \multirow{2}{*}{$<0.0001$} \\
\hline Negative & $2(1)$ & $77(48)$ & \\
\hline DLNs [mean (range, SD)] & $23.9(12-85,14.4)$ & $22.4(12-91,11.1)$ & 0.68 \\
\hline MLNs [mean (range, SD)] & $5.51(2-18,3.55)$ & $2.19(1-13,1.95)$ & $<0.0001$ \\
\hline LNR [median (IQR)] & $0.24(0.13-0.37)$ & $0.08(0.05-0.14)$ & $<0.0001$ \\
\hline \multicolumn{4}{|l|}{ Operative approach $[n(\%)]$} \\
\hline Open & $21(13)$ & $62(39)$ & \multirow[t]{2}{*}{0.016} \\
\hline Laparoscopic & $8(5)$ & $68(43)$ & \\
\hline \multicolumn{4}{|l|}{ Adjuvant chemotherapy $[n(\%)]$} \\
\hline With & $22(14)$ & $85(53)$ & \multirow[t]{2}{*}{0.28} \\
\hline Without & 7 (4) & $45(28)$ & \\
\hline
\end{tabular}

Furthermore, in the subgroup analysis for the 128 patients with $<5$ MLNs, the 5-year OS rates of the patients with $\geq 2$ ( $n=$ $14)$ and $<2$ MLNs-PDCs $(n=114)$ were 68 and $88 \%(p=$ 0.037 ). In contrast, in subgroup analysis for the 26 patients with $\geq 5 \mathrm{MLNs}$, there was no significant difference in the 5year OS rates between the patients with $\geq 2$ and $<2$ MLNsPDCs (59 and $53 \% ; p=0.70)$. In addition, in another subgroup analysis for patients who received adjuvant chemotherapy and those who did not, 5-year OS rates of patients with and without chemotherapy were 67 vs. $64 \%$ in $\geq 2$ MLNsPDCs and 87 vs. $82 \%$ in $<2$ MLNs-PDCs, and no significant difference was found between the groups.

As Table 3 shows, among the representative clinicopathological variables, including LN status, the following five variables were identified using the AIC in multivariate analysis: tumor location (rectum vs. colon), number of MLNs-PDCs $(\geq 2$ vs. $<2$ ), sex (female vs. male), tumor size ( $\geq 50$ vs. $<50 \mathrm{~mm}$ ), and T status (pT4 vs. pT1-3). Consequently, both pT4 tumors $(\mathrm{HR}=3.54, p<0.001)$ and $\geq 2$ MLNs-PDCs $(\mathrm{HR}=$ $2.50, p=0.038)$ were revealed as significant prognosticators for 5-year OS. Finally, the prognostic model incorporating $\geq 2$ MLNs-PDCs had higher accuracy with lower AIC value
$(\mathrm{AIC}=230.08)$ than models incorporating other indicators of LN status, such as $\geq 5$ MLNs $(\mathrm{AIC}=231.21)$ or $\geq 30 \% \mathrm{LNR}$ $(\mathrm{AIC}=231.77)$.

\section{Discussion}

The present study examined prognostic properties of the number of MLNs-PDCs in a retrospective cohort comprising 159 patients with pStage III CRC. Since the clinical importance of PDCs in primary tumors has been clarified successfully [18, $19,23,26]$, criteria modified by the authors was applied to investigate histopathological features in MLNs. To our knowledge, this is the first study to highlight the histopathological features of PDCs in MLNs as a candidate for a new LN status.

Results showed that $\geq 2$ MLNs-PDCs were associated closely with PDC-positive primary tumors, implying that PDC status in metastatic sites frequently could parallel that in primary tumors. On the other hand, this study found that some PDC-positive tumors were not accompanied by PDCpositive MLNs. Using similar approaches, Takahashi et al. [27] found histopathological heterogeneity between primary 
Table 3 Univariate and multivariate survival analysis of histological valuables

\begin{tabular}{lllll}
\hline Variables & Univariate & & \\
& & & & Multivariate $^{\mathrm{b}}$ \\
\cline { 3 - 5 } & $p$ Value & HR & $95 \%$ CI & $p$ Value \\
\hline Age (year) $(\geq 65$ vs. $<65)$ & 0.29 & - & - & - \\
Sex (female vs. male) & 0.23 & 2.15 & $0.88-5.24$ & 0.093 \\
Tumor location (rectum vs. colon) & 0.072 & 2.23 & $0.98-5.05$ & 0.055 \\
Tumor size $(\geq 50$ vs. $<50$ mm) & 0.70 & 2.20 & $0.89-5.45$ & 0.089 \\
T status (T4 vs. T1-3) & 0.032 & 3.54 & $1.37-9.14$ & 0.009 \\
MLNs $(\geq 5$ vs. $<5)$ & 0.024 & - & - & - \\
LNR $(\geq 30$ vs. $<30 \%)$ & 0.004 & - & - & - \\
MLNs-PDCs $(\geq 2$ vs. $<2)$ & 0.008 & 2.50 & $1.09-5.74$ & 0.031 \\
Adjuvant chemotherapy (without vs. with) & 0.31 & - & - & - \\
\hline
\end{tabular}

${ }^{a}$ Kaplan and Meier method, and statistical significance was determined by log-rank test

${ }^{\mathrm{b}}$ Multivariate survival analysis was performed using Cox's proportional hazard model

$H R$ hazard ratio, $C I$ confidence interval tumors and their synchronous MLNs in CRC patients. Furthermore, it is worth mentioning that Mori et al. [28] demonstrated that histopathological heterogeneity between primary tumors and their synchronous MLNs was associated with poorer survival even after adjuvant chemotherapy was administered. However, in the subgroup of this study that examined the effect of adjuvant chemotherapy, no significant difference was found between patients with $\geq 2$ and $<2$ MLNs-PDCs. It is possible that this inconsistent result is due to the small sample size. Another possible explanation is that there was no control over the chemotherapy regimens given to patients, since those regimens progressed from single-dose 5 -FU to combinations using 5 -FU and oxaliplatin or irinotecan or both $[2,3]$ over the period of this study. In this context, additional prospective studies using larger sample sizes would confirm our results.

The most important finding of this study was that $\geq 2$ MLNs-PDCs were associated strongly with a poorer 5-year OS. Although the number of MLNs-PDCs was correlated moderately positively with the number of MLNs, the number of MLNs-PDCs could provide the highest degree of discrimination regarding patient survival compared with the number of MLNs and other measures of LN status that are based on the theory of AIC. These findings suggest that the number of MLNs-PDCs could provide more precise information on potential tumor aggressiveness at the metastatic site. Furthermore, this study assessed the subgroups of patients with $<5 \mathrm{MLNs}$, and there was significant difference in the 5year OS rates between patients with $\geq 2$ and $<2$ MLNs-PDCs. Consequently, this outcome suggests that MLNs-PDCs might contribute to further survival stratification, especially for CRC patients with $<5$ MLNs. Interestingly, Komuta et al. [29] and Fujii et al. [30] reported the "extracapsular invasion" of MLNs, which was highly indicative of tumor aggressiveness at the metastatic site. This concept seems to be similar to that of PDCs in MLNs in our study. Likewise, Ueno et al. [16] described EX that exhibited active, invasive lesions as "aggressive EX," which was associated with a dramatically worse prognosis. Since evaluation criteria in this study allowed EX to be comprehensively considered as MLNs, it is possible that MLNs with positive PDCs included some aggressive EX.

This study has some potential limitations. First, the 5-year OS rate of $79 \%$ in patients with pStage III CRC in this cohort seems to be relatively better than that estimated in studies with larger populations $[2,3]$. The reason for this remains unclear. Second, the exceedingly high hazard ratio may be a product of the single-institution experience and the relatively small sample size in this study. Another question is why $\geq 2$ MLNsPDCs were related to open surgery $(p=0.016)$ even though the number of DLNs and MLNs did not differ statistically between open and laparoscopic surgery. One possible explanation is surgeons' preoperative choices, in which cases with clinically diagnosed T4 tumors were treated by open surgery. In addition, although cumulative evidence has confirmed a larger number of MLNs as a strong prognosticator of CRC, cutoff thresholds for some LN statuses addressed in this study remain under intense discussion. With respect to the cutoff threshold of LNR, Yuan et al. [13] defined it as $33 \%$, Gleisner et al. [14] as $50 \%$, and Madbouly et al. [15] as $38 \%$. Although consensus has not been reached regarding this cutoff, they agreed that a higher LNR was an adverse prognosticator. The cutoff of $30 \%$ in this study seems to be comparable to aforementioned data. In contrast, similar to the experience of Kotake et al. [10], we did not identify a statistically reliable cutoff for the number of DLNs. Although existing guidelines $[5,6]$ recommend that $\geq 12$ DLNs be examined to eliminate possible staging migration, some researchers 
claim that there is no minimum number of DLNs that would enhance the accuracy of staging pStage III CRC $[8,9]$. Therefore, this issue merits further discussion.

\section{Conclusion}

The PDCs in MLNs were closely associated with that in primary tumors. The number of MLNs-PDCs could exert an effect on survival and provide more concise information to improve stratification in pStage III CRC and, consequently, could be a candidate for a new, alternative LN status. This study demonstrated the need for special observation of PDCs in MLNs during pathological diagnosis.

Acknowledgments We would like to express appreciation to the technical staff members in our institution. In particular, the authors would like to thank A. Yoshimura, H. Yuki, and T. Takabayashi for their excellent technical assistance.

Conflict of interest We received no funding or grant support for this study.

Open Access This article is distributed under the terms of the Creative Commons Attribution 4.0 International License (http:// creativecommons.org/licenses/by/4.0/), which permits unrestricted use, distribution, and reproduction in any medium, provided you give appropriate credit to the original author(s) and the source, provide a link to the Creative Commons license, and indicate if changes were made.

\section{References}

1. Ferlay J, Shin HR, Bray F et al (2010) Estimates of worldwide burden of cancer in 2008: GLOBOCAN 2008. Int J Cancer 127: 2893-2917. doi:10.1002/ijc. 25516

2. Hurwitz H, Fehrenbacher L, Novotny W et al (2004) Bevacizumab plus irinotecan, fluorouracil, and leucovorin for metastatic colorectal cancer. N Engl J Med 350:2335-2342

3. Fuchs CS, Marshall J, Mitchell E et al (2007) Randomized, controlled trial of irinotecan plus infusional, bolus, or oral fluoropyrimidines in first-line treatment of metastatic colorectal cancer: results from the BICC-C Study. J Clin Oncol 25:47794786. doi: $10.1200 /$ JCO.2007.15.5390

4. Dukes C (1937) Histological grading of rectal cancer: (section of pathology). Proc R Soc Med 30:371-376

5. Leslie HS, Mary KG, Christian W (2009) TNM classification of malignant tumours, 7th edition, Wiley-Blackwell

6. Japanese Society for Cancer of the Colon and Rectum (2013) Japanese classification for colorectal carcinoma, 8th edn. Kanehara-shuppan, Tokyo

7. Tepper JE, O'Connell MJ, Niedzwiecki D (2001) Impact of number of nodes retrieved on outcome in patients with rectal cancer. J Clin Oncol 19:157-163

8. Goldstein NS (2002) Lymph node recoveries from 2427 pT3 colorectal resection specimens spanning 45 years: recommendations for a minimum number of recovered lymph nodes based on predictive probabilities. Am J Surg Pathol 26:179-189

9. Chou JF, Row D, Gonen M et al (2010) Clinical and pathologic factors that predict lymph node yield from surgical specimens in colorectal cancer: a population-based study. Cancer 116:2560 2570. doi: $10.1002 /$ cncr.25032

10. Kotake K, Honjo S, Sugihara K et al (2012) Number of lymph nodes retrieved is an important determinant of survival of patients with stage II and stage III colorectal cancer. Jpn J Clin Oncol 42:29 35. doi:10.1093/jjco/hyr164

11. Berger AC, Sigurdson ER, LeVoyer T et al (2005) Colon cancer survival is associated with decreasing ratio of metastatic to examined lymph nodes. J Clin Oncol 23:8706-8712

12. Kobayashi H, Mochizuki H, Kato T et al (2011) Lymph node ratio is a powerful prognostic index in patients with stage III distal rectal cancer: a Japanese multicenter study. Int J Colorectal Dis 26:891896. doi:10.1007/s00384-011-1173-0

13. Yuan Y, Li MD, Hu HG et al (2013) Prognostic and survival analysis of 837 Chinese colorectal cancer patients. World J Gastroenterol 19:2650-2659. doi:10.3748/wjg.v19.i17.2650

14. Gleisner AL, Mogal H, Dodson R et al (2013) Nodal status, number of lymph nodes examined, and lymph node ratio: what defines prognosis after resection of colon adenocarcinoma? J Am Coll Surg 217:1090-1100

15. Madbouly KM, Abbas KS, Hussein AM (2014) Metastatic lymph node ratio in stage III rectal carcinoma is a valuable prognostic factor even with less than 12 lymph nodes retrieved: a prospective study. Am J Surg 207:824-831. doi:10.1016/j.amjsurg.2013.07.022

16. Ueno H, Mochizuki H, Hashiguchi Y et al (2007) Extramural cancer deposits without nodal structure in colorectal cancer: optimal categorization for prognostic staging. Am J Clin Pathol 127:287294

17. Ueno H, Mochizuki H, Shirouzu K et al (2012) Multicenter study for optimal categorization of extramural tumor deposits for colorectal cancer staging. Ann Surg 255:739-746. doi:10.1097/SLA. 0b013e31824b4839

18. Ueno H, Kajiwara Y, Shimazaki H et al (2012) New criteria for histologic grading of colorectal cancer. Am J Surg Pathol 36:193201. doi:10.1097/PAS.0b013e318235edee

19. Ueno H, Hase K, Hashiguchi $Y$ et al (2014) Site-specific tumor grading system in colorectal cancer: multicenter pathologic review of the value of quantifying poorly differentiated clusters. Am J Surg Pathol 38:197-204. doi:10.1097/ PAS.0000000000000113

20. Barresi V, Branca G, Vitarelli E et al (2014) Micropapillary pattern and poorly differentiated clusters represent the same biological phenomenon in colorectal cancer: a proposal for a change in terminology. Am J Clin Pathol 142:375-383. doi:10.1309/ AJCPFEA7KA0SBBNA

21. Sugihara K, Kobayashi H, Kato Tet al (2006) Indication and benefit of pelvic sidewall dissection for rectal cancer. Dis Colon Rectum 49:1663-1672

22. Akiyoshi T, Watanabe T, Miyata S et al (2012) Results of a Japanese nationwide multi-institutional study on lateral pelvic lymph node metastasis in low rectal cancer: is it regional or distant disease? Ann Surg 255:1129-1134. doi:10.1097/ SLA.0b013e3182565d9d

23. Kinoshita O, Kishimoto M, Murayama Y et al (2015) Semiquantified pathological evaluation of poorly differentiated clusters in non-mucinous pT2-3 colorectal carcinoma. J World Surg Oncol 13:140. doi:10.1186/s12957-015-0550-5

24. Akaike H (1974) A new look at the statistical model identification. IEEE Trans Autom Control 19:716-723

25. Kanda Y (2013) Investigation of the freely available easy-to-use software 'EZR' for medical statistics. Bone Marrow Transplant 48: 452-458. doi:10.1038/bmt.2012.244

26. Barresi V, Bonetti LR, Ieni A et al (2014) Histologic grading based on counting poorly differentiated clusters in preoperative biopsy predicts nodal involvement and pTNM stage in colorectal cancer 
patients. Hum Pathol 45:268-275. doi:10.1016/j.humpath.2013.07. 046

27. Takahashi K, Mori T, Yasuno M (2000) Histologic grade of metastatic lymph node and prognosis of rectal cancer. Dis Colon Rectum 43:S40-6

28. Mori T, Hirota T, Ohashi Y et al (2006) Significance of histologic type of primary lesion and metastatic lymph nodes as a prognostic factor in stage III colon cancer. Dis Colon Rectum 49:982-992
29. Komuta K, Okudaira S, Haraguchi M et al (2001) Identification of extracapsular invasion of the metastatic lymph nodes as a useful prognostic sign in patients with resectable colorectal cancer. Dis Colon Rectum 44:1838-1844

30. Fujii T, Tabe Y, Yajima R et al (2011) Process of distant lymph node metastasis in colorectal carcinoma: implication of extracapsular invasion of lymph node metastasis. BMC Cancer 11:216. doi:10. 1186/1471-2407-11-216 